09

\title{
Поляритонные возбуждения в неидеальной двухподрешеточной цепочке микропор
}

\author{
(C) В.В. Румянцев, С.А. Федоров, К.В. Гуменник, Ю.А. Паладян \\ Донецкий фризико-технический институт им. А.А. Галкина, \\ 83114 Донецк, Украина \\ e-mail: 380957931135@yandex.ru
}

Поступило в Редакцию 18 апреля 2019 г.

В окончательной редакции 27 мая 2019 г.

Принято к публикации 5 ноября 2019 г.

Исследована зависимость дисперсионных характеристик поляритонных возбуждений в неидеальной $1 D$ решетке микропор от концентрации дефектов структуры, связанных с вариацией положений микропор, содержащих в одной из подрешеток квантовые точки. Показано, что в результате наличия данных структурных дефектов в $1 D$-двухподрешеточном массиве микропор можно добиться необходимого изменения энергетической структуры поляритонных возбуждений и, следовательно, оптических свойств исследуемой системы, обусловленных перестройкой электромагнитного спектра.

Ключевые слова: неидеальная $1 D$-решетка микропор-резонаторов, поляритонные возбуждения, квантовые точки, приближение виртуального кристалла.

DOI: $10.21883 / J T F .2020 .05 .49189 .168-19$

\section{Введение}

Среди задач, которые приходится решать при создании новых материалов используемых в качестве источников когерентного излучения, и устройств, работающих на их основе, встает проблема изучения особых структур поляритонных кристаллов [1], представляющих собой отдельный класс фотонных кристаллов [2]. В поляритонных структурах реализуется сильная связь квантовых возмушений (экситонов) среды и оптического поля. В процессе изучения поляритонных кристаллов рождается новая область науки - поляритоника [3], как самостоятельный раздел фотоники.

В частности, поляритонной структурой может быть пространственно-периодическая система связанных микропор (резонаторов) [4,5]. Интерес к изучению оптических мод в системе микрорезонаторов, который появился в связи с созданием оптоэлектронных устройств [6], значительно вырос в последнее время. Здесь стоит отметить резонаторы на основе дефектов в фотонных кристаллах $[7,8]$, наличие сильной связи между квантовой точкой и таким микрорезонатором было продемонстрировано в [9]. Теоретическое исследование формирования в цепочке микрорезонаторов квантовых солитонов, связанных с поляритонами нижней дисперсионной ветви (НДВ), выполнено в работах $[1,9]$. Авторы $[1,9]$ полагают, что микрорезонаторы могут быть привлекательны для целей квантовой обработки информации. Прогресс, достигнутый в последнее время в плане создания высокодобротных полупроводниковых микрорезонаторов с брэгговскими зеркалами, позволил получить и исследовать бозе-эйнштейновскую конденсацию, а также сверхтекучие свойства НДВ поляритонов в квантовых ямах, внедренных в полупроводниковые (CdTe/CdMgTe или GaAs) структуры микрорезонаторов [10-12]. Поляритоны в этих системах рассматриваются как термодинамически квазиравновесный двумерный газ взаимодействующих бозонных частиц.

На основе развитых авторами ранее представлений о неидеальных одномерных поляритонных структуpax $[3,7,8]$ в настоящей работе рассмотрены электромагнитные возбуждения в неидеальной двухподрешеточной цепочке микропор (резонаторов) с переменным периодом, причем система туннельно-связанных микрорезонаторов содержит в одной из подрешеток в качестве атомарной подсистемы квантовые точки. Ниже изучены особенности дисперсии поляритонных возбуждений в неидеальной $1 D$-решетке связанных микропор-резонаторов, вызванные случайными вариациями их положений.

\section{Теоретическая модель}

Опираясь на разработанный в $[3,7,8,13]$ подход, рассмотрим электромагнитные возбуждения в неидеальной двухподрешеточной цепочке микропор (резонаторов) с переменным периодом $d=a_{1}^{v}+a_{2}^{u}$. Здесь $a_{1}^{v}+a_{2}^{\mu}-$ варьируемые положения микропор соответственно в первой и второй подрешетках. В данном случае нижний индекс (1 или 2) - номер подрешетки, верхние индексы $(\nu, \mu)$ обозначают положение соответствующего резонатора в ячейке, которые также могут принимать значения 1 или 2. Каждый из туннельно-связанных микрорезонаторов содержит по одной оптической моде. В общем случае квадратичная часть гамильтониана $\hat{H}^{e x}$, описывающая элементарные возбуждения в цепочке микропор (как содержащих квантовые точки, так и без них) в предположении, что плотность возбужденных 
состояний структурных элементов в резонаторной и атомарной подсистемах мала, в приближении Гайтлера-Лондона [14] в одноуровневой модели имеет вид:

$$
\hat{H}^{e x}=\sum_{\alpha, \beta=1}^{2} \sum_{\substack{n, m, \lambda, \sigma}} D_{n \alpha, m \beta}^{\lambda \sigma} \hat{\Phi}_{n \alpha \lambda}^{+} \hat{\Phi}_{m \beta \sigma}
$$

где $n, m-$ номера элементарных ячеек, $\alpha, \beta-$ номера подрешеток.

$$
\begin{gathered}
D_{n \alpha, m \beta}^{11}=h \omega_{n \alpha}^{a t} \delta_{n \alpha, m \beta}+V_{n \alpha, m \beta}, \\
D_{n \alpha, m \beta}^{22}=h \omega_{n \alpha}^{p h} \delta_{n \alpha, m \beta}-A_{n \alpha, m \beta}, \\
D_{n \alpha, m \beta}^{12}=D_{n \alpha, m \beta}^{21}=g_{n a} \delta_{n \alpha, m \beta}, \hat{\Phi}_{n \alpha}^{\lambda=2}=\hat{\Psi}_{n \alpha}, \hat{\Phi}_{n \alpha}^{\lambda=1}=\hat{B}_{n \alpha} .
\end{gathered}
$$

В выражениях (1) и (2) в узельном представлении: $\omega_{n \alpha}^{p h}$ - частота фотонной моды электромагнитного возбуждения, локализованного в $n \alpha$-ом узле (резонаторе), $\hat{\Psi}_{n \alpha}^{+}, \hat{\Psi}_{n \alpha}$ - бозе-операторы рождения и уничтожения этой фотонной моды, $h \omega_{n \alpha}^{a t}-$ энергия возбуждения квантовой точки в узле $n \alpha, \hat{B}_{n \alpha}, \hat{B}_{n \alpha}^{+}-$бозе-операторы рождения и уничтожения этого возбуждения, $A_{n \alpha m \beta}-$ матрица резонансного взаимодействия, характеризующая перекрытие оптических полей резонаторов $n \alpha$-го и $m \beta$-го узлов решетки и, следовательно, определяющего вероятность перескока соответствующего электромагнитного возбуждения, $V_{n \alpha m \beta}$ - матрица резонансного взаимодействия квантовых точек в узлах $n \alpha$ и $m \beta, g_{n \alpha}-$ матрица резонансного взаимодействия квантовой точки в узле $n \alpha$ с локализованным в этом узле электромагнитным полем. Индексы $\lambda, \sigma$ фиксируют наличие (при значении 1) или отсутствие (при значении 2) квантовой точки в соответствующей микропоре.

Расчет поляритонного спектра $\Omega(k)$ рассматриваемой системы проведем, используя аппарат усредненных функций Грина, в приближении виртуального кристалла $[15,16]$. В указанном приближении усредненная резольвента квазичастичного гамильтониана системы равна резольвенте усредненного гамильтониана. Поэтому величины $D_{n \alpha, m \beta}^{\lambda \sigma}$ в равенстве (1) следует заменить на их конфигурационно усредненные значения $\left(D_{n \alpha, m \beta}^{\lambda \sigma} \rightarrow\left\langle D_{n \alpha, m \beta}^{\lambda \sigma}\right\rangle\right)$ по всем возможным вариациям положений микропор (процедура конфигурационного усреднения массива микропор обозначена угловыми скобками). Такая операция „восстанавливает трансляционную инвариантность и позволяет перейти в $k$-представление с последующей диагонализацией гамильтониана путем применения процедуры Боголюбова-Тябликова [14]. Выполнение указанных процедур позволяет получить следующее уравнение для нахождения $\Omega(k)$ :

$$
\operatorname{det}\left\|D_{\alpha \beta}^{\lambda \sigma}(k)-h \Omega(k) \delta_{\alpha \beta} \delta_{\lambda \sigma}\right\|=0
$$

где

$$
D_{\alpha \beta}^{\lambda \sigma}(k)=\sum_{m}\left\langle D_{n \alpha m \beta}^{\lambda \sigma}\right\rangle \exp \left[i k\left(r_{n \alpha}-r_{m \beta}\right)\right]
$$

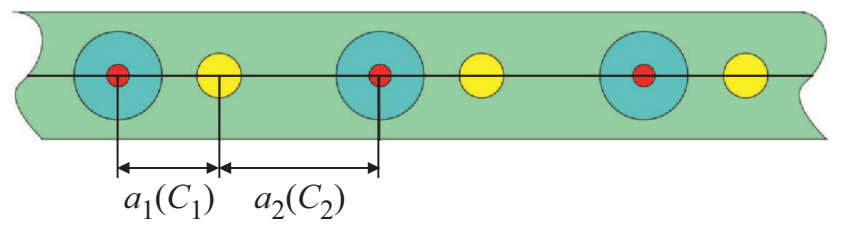

Рис. 1. Схематическое изображение усредненного неидеального двухподрешеточного $1 D$-массива микропор, первая подрешетка которого содержит одинаковые квантовые точки.

Заметим, что волновое число $k$, характеризующее собственные состояния электромагнитных возбуждений в исследуемой системе, изменяется в пределах первой зоны Бриллюэна для виртуальной решетки с периодом

$$
\begin{aligned}
\langle d\rangle & =\left\langle a_{1}^{\mu}\right\rangle+\left\langle a_{2}^{\nu}\right\rangle \\
& =C_{1}^{(1)} a_{1}^{(1)}+C_{1}^{(2)} a_{1}^{(2)}+C_{2}^{(1)} a_{2}^{(1)}+C_{2}^{(2)} a_{2}^{(2)},
\end{aligned}
$$

где $C_{1(2)}^{1(2)}-$ концентрации соответствующих положений микропор $a_{1}^{v}, a_{2}^{\mu}$. Причем $C_{1}^{(1)}+C_{1}^{(2)}=1, C_{2}^{(1)}+C_{2}^{(2)}=1$, следовательно, $C_{1}^{(2)}=1-C_{1}^{(1)} \equiv C_{1}, C_{2}^{(2)}=1-C_{2}^{(1)} \equiv C_{2}$. Таким образом, $\langle d\rangle=a_{1}\left(C_{1}\right)+a_{2}\left(C_{2}\right) \equiv d\left(C_{1}, C_{2}\right)$, где $a_{1}\left(C_{1}\right)=a_{1}^{(1)}+\left(a_{1}^{(2)}-a_{1}^{(1)}\right) C_{1}, \quad a_{2}\left(C_{2}\right)=a_{2}^{(1)}+\left(a_{2}^{(2)}-\right.$ $\left.-a_{2}^{(1)}\right) C_{2}$.

\section{Результаты и обсуждение}

Рассмотрим подробнее электромагнитные возбуждения в двухподрешеточной $1 D$-виртуальной решетке микропор (рис. 1), содержащих квантовые точки лишь одного сорта в одной из подрешеток (например, в первой, т.е. $\alpha=\beta=1)$. Положение микрорезонатора определяет равенство: $r_{n \alpha}=r_{n}+r_{\alpha}$. Поэтому в нашем случае положение микропор первой и второй подрешеток в нулевой элементарной ячейке $\left(r_{n=0}=0\right)$ задают соотношения соответственно: $r_{01}=0$ и $r_{02}=a_{1}\left(C_{1}\right)=d\left(C_{1}, C_{2}\right)-a_{2}\left(C_{2}\right)$. Спектр поляритонных возбуждений $\Omega(k)$ в приближении ближайших соседей следует из соотношений (2) и (3):

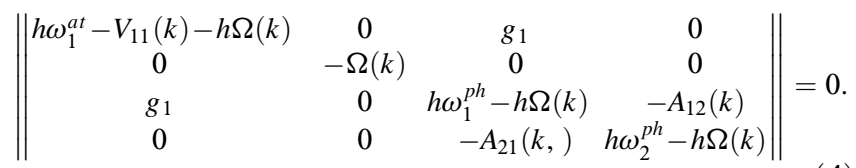

Фурье-образ матрицы $\left\langle V_{n 1 m 1}\right\rangle$ резонансного взаимодействия квантовых точек в узлах $n 1$ и $m 1$ в приближении ближайших соседей имеет вид $V_{11}(k)=V_{11}\left(k, C_{1}, C_{2}\right) \cong$ $2 V_{11}\left[d\left(C_{1}, C_{2}\right)\right] \cos \left[k d\left(C_{1}, C_{2}\right)\right]$, где $V_{11}\left[d\left(C_{1}, C_{2}\right)\right]$ полагаем равным $V_{11}\left[d\left(C_{1}, C_{2}\right)\right]=V_{11}[d(0)] \exp \left[-\frac{\left|d\left(C_{1}, C_{2}\right)-d(0)\right|}{d(0)}\right]$. Поскольку квантовые точки при данном рассмотрении по составу не варьируются, параметр резонансного взаимодействия квантовой точки в любом узле с локализованным в этом узле электромагнитным полем равен $g_{1}$ 

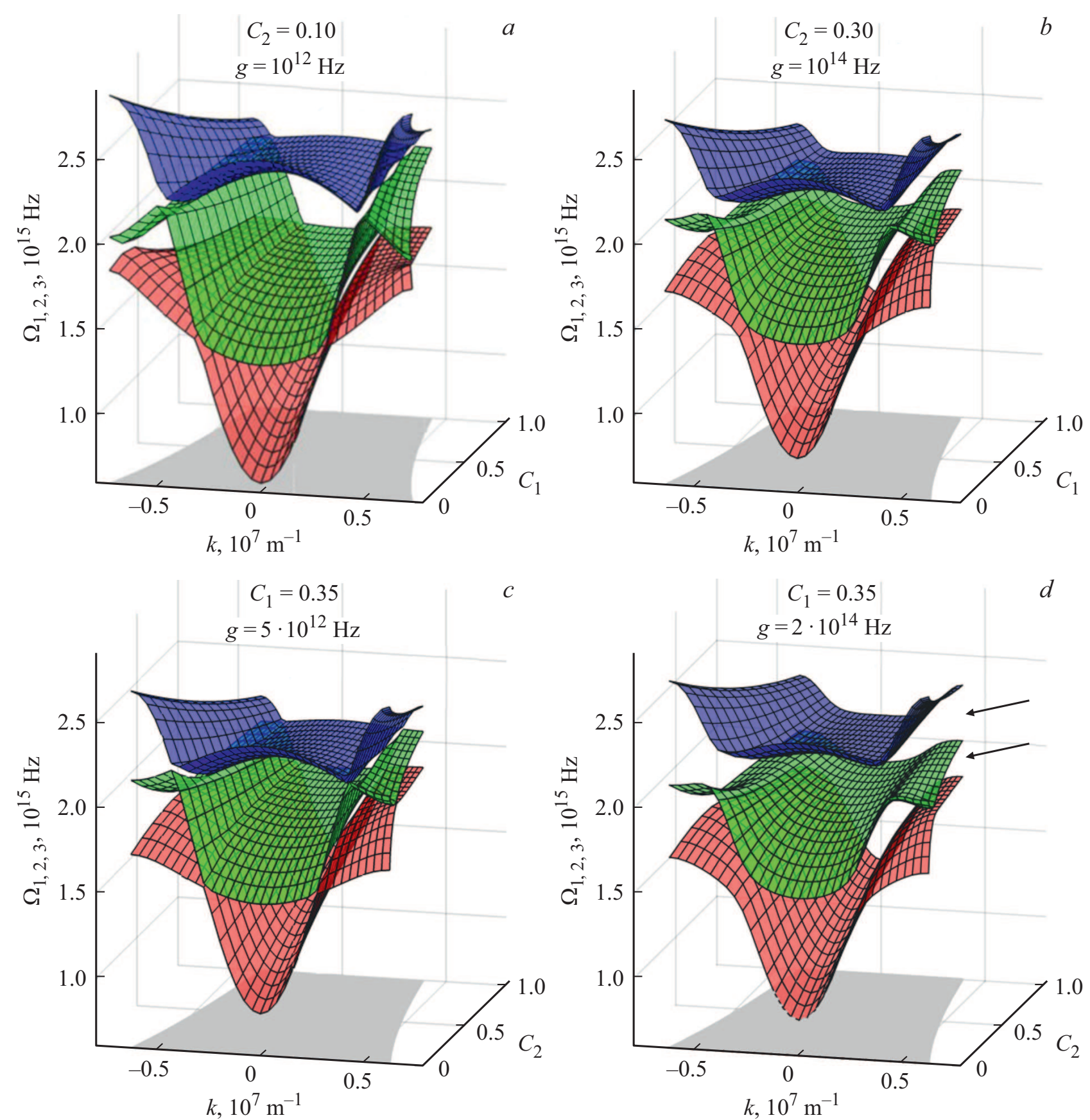

Рис. 2. Зависимость дисперсии поляритонов $\Omega_{1,2,3}\left(k, C_{1}, C_{2}\right)$ от концентрации дефектов структуры для различных значений параметра $g \equiv g_{1} / h$ резонансного взаимодействия квантовой точки в узле с локализованным в этом узле электромагнитным полем (стрелки указывают на изменение величины „бутылочного горла“ в зависимости от величины $g$ ).

(не зависит от $n$ ). Величины $A_{\alpha \beta}(k) \equiv A_{\alpha \beta}\left(k, C_{1}, C_{2}\right)$ в (4) - фурье-образы матрицы $\left\langle A_{n \alpha m \beta}\right\rangle$ резонансного взаимодействия:

$$
A_{\alpha \beta}(k)=\sum_{m}\left\langle A_{n \alpha m \beta}\right\rangle \exp \left[i k\left(r_{n \alpha}-r_{m \beta}\right)\right]
$$

В рамках используемой модели в приближении ближайших соседей фурье-образы $A_{\alpha \beta}\left(k, C_{1}, C_{2}\right)$ приобретают следующий вид:

$$
\begin{aligned}
A_{12}\left(k, C_{1}, C_{2}\right) \cong & A_{12}\left[a_{1}\left(C_{1}\right)\right] \exp \left[-i k a_{1}\left(C_{1}\right)\right] \\
& +A_{12}\left[a_{2}\left(C_{2}\right)\right] \exp \left\{i k\left[a_{2}\left(C_{2}\right)\right]\right\}, \\
A_{21}\left(k, C_{1}, C_{2}\right) \cong & A_{21}\left[a_{1}\left(C_{1}\right)\right] \exp \left[i k a_{1}\left(C_{1}\right)\right] \\
& +A_{21}\left[a_{2}\left(C_{2}\right)\right] \exp \left\{-i k\left[a_{2}\left(C_{2}\right)\right]\right\},
\end{aligned}
$$



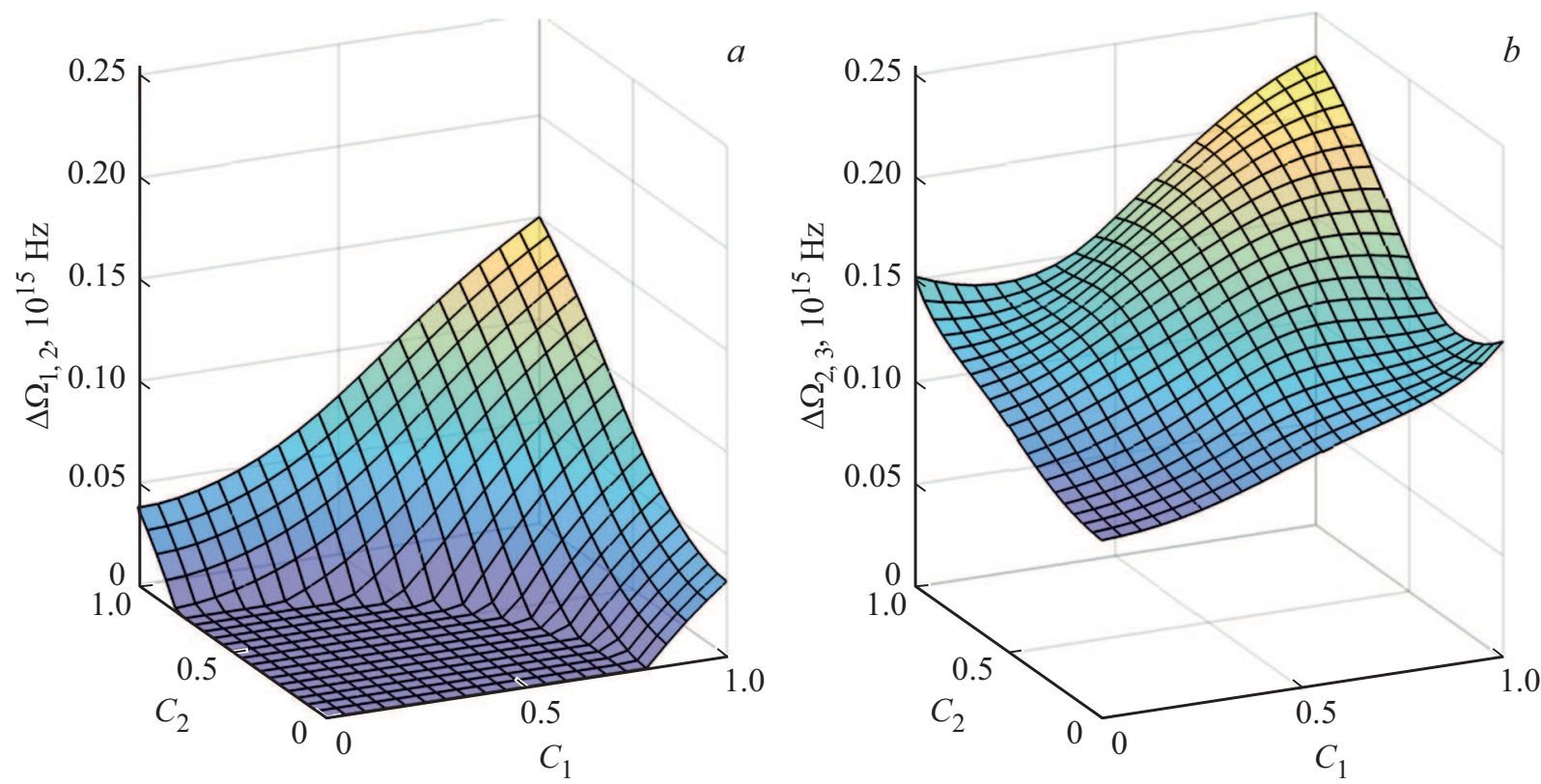

Рис. 3. Зависимость $\Delta \Omega_{12(23)}\left(C_{1}, C_{2}\right)$ ширины соответствующей запрещенной зоны от концентрации дефектов исследуемой структуры.

Величины $A_{12(21)}\left[a_{1(2)}\left(C_{1(2)}\right)\right]$, являющиеся компонентами усредненной матрицы $\left\langle A_{n \alpha m \beta}\right\rangle$ резонансного взаимодействия, полагаем вслед за [7] равными

$$
\begin{aligned}
A_{12(21)} & {\left[a_{1(2)}\left(C_{1(2)}\right)\right]=A_{12(21)}\left[a_{1(2)}(0)\right] } \\
& \times \exp \left[-\frac{\left|a_{1(2)}\left(C_{1(2)}\right)-a_{1(2)}(0)\right|}{a_{1(2)}(0)}\right] .
\end{aligned}
$$

В рамках используемой модели $A_{12} \simeq A_{21} \gg A_{11}, A_{22}$. Соотношение (4) показывает, что закон дисперсии $\Omega(k)$ элементарных электромагнитных возбуждений в искомой системе микропор определяется как частотными характеристиками решетки резонаторов, так и величинами $A_{\alpha \beta}(k)$.

Дальнейшие численные расчеты выполнены в среде компьютерного пакета Matlab, который включает в себя язык инженерного программирования с использованием программ, входящих в стандартную библиотеку Matlab. В частности, для численного отыскания корней кубического уравнения относительно частоты $\Omega$, возникающего при раскрытии детерминанта (6), применялась программа из библиотеки Matlab fzero.m, в основе которой лежит итерационный алгоритм Ньютона.

При численном моделировании соответствующих параметров, определяющих особенности спектра электромагнитных возбуждений, в настоящей работе взяты значения величин того же порядка, что и используемые авторами ранее при исследовании подобных структур $[7,8]$. В частности, полагаем, что частота возбуждения квантовой точки в узле равна $\omega_{2}^{a t}=2 \pi \cdot 202 \mathrm{THz}$ (в рамках используемого приближения Гайтлера-Лондона $\left.h \omega^{a t} \gg V_{11}\right)$, значения частот резонансных фотонных мод, локализованных в микропорах решетки, $\omega_{1}^{p h}=2 \pi \cdot 311 \mathrm{THz}$ и $\omega_{2}^{p h}=2 \pi \cdot 331 \mathrm{THz}$. Параметры, определяющие перекрытие оптических полей и взаимодействие квантовых точек в соседних микропорах, взяты следующими: $A_{12}\left[a_{1}(0)\right] / 2 \hbar=3.5 \cdot 10^{14} \mathrm{~Hz}$, $A_{12}\left[a_{2}(0)\right] / 2 \hbar=1.2 \cdot 10^{14} \mathrm{~Hz}, V_{11} / 2 \hbar=1 \cdot 10^{13} \mathrm{~Hz}$, причем $a_{1}(0)=a_{1}^{(1)}=1 \cdot 10^{-7} \mathrm{~m}, a_{2}(0)=a_{2}^{(1)}=3 \cdot 10^{-7} \mathrm{~m}$, $d(0)=a_{1}(0)+a_{2}(0)$. Поверхности, описывающие дисперсионную зависимость частот $\Omega_{1,2,3}\left(k, C_{1}, C_{2}\right)$, исследуемых коллективных возбуждений в неидеальной решетке микрорезонаторов, содержащих в одной из подрешеток квантовые точки, представлены на рис. 1. При этом $k$ изменяется в пределах первой зоны Бриллюэна: $-\frac{\pi}{d\left(C_{1}, C_{2}\right)}<k<\frac{\pi}{d\left(C_{1}, C_{2}\right)}$ (затемненная область $\left.k, C_{1(2)}\right)$ на рис. 2). Заметим, что форма дисперсионной поверхности $\Omega_{3}\left(k, C_{1}, C_{2}\right)$ на рис. 2 свидетельствует о возможности существования при определенных концентрациях дефектов структуры бозе-эйнштейновского поляритонного конденсата благодаря наличию локального минимума при значениях $k \neq 0$ (дополнительно к возможности существования конденсата при $k=0$, на которую указывают поверхности $\left.\Omega_{1,2}\left(k, C_{1}, C_{2}\right)\right)$. Зависимость ширины соответствующей запрещенной зоны поляритонного спектра $\Delta \Omega_{12(23)}\left(C_{1}, C_{2}\right) \equiv \min _{k}\left[\Omega_{2(3)}\left(C_{1}, C_{2}\right)-\Omega_{1(2)}\left(C_{1}, C_{2}\right)\right]$ от концентрации дефектов исследуемой структуры приведена на рис. 3.

Немонотонный характер концентрационной зависимости $\Omega_{1,2,3}\left(k, C_{1}, C_{2}\right)$ поляритонного спектра таких неидеальных систем проявляется в особенностях зависимости эффективной массы

$$
m_{\mathrm{eff}}^{1,2,3}\left(C_{1}, C_{2}\right) \equiv \hbar\left(\left.\frac{\partial^{2} \Omega_{1,2,3}\left(k, C_{1}, C_{2}\right)}{\partial k^{2}}\right|_{k=0}\right)^{-1}
$$



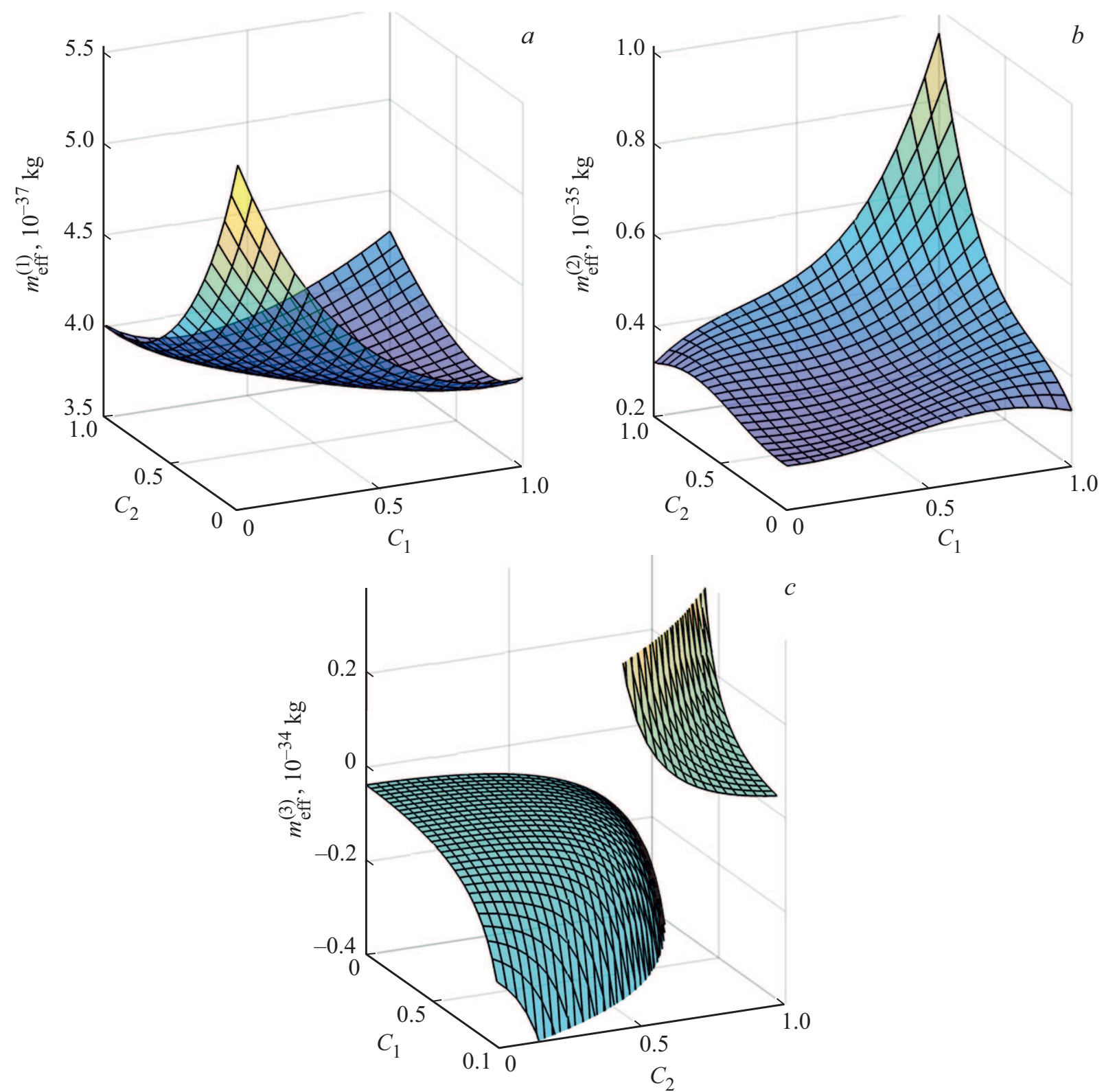

Рис. 4. Зависимость эффективной массы $m_{\text {eff }}^{1,2,3}\left(C_{1}, C_{2}\right)$ поляритонных возбуждений от концентрации дефектов в $1 D$ неидеальной решетке связанных микропор.

исследуемых квазичастиц (рис. 4). Последнее дает дополнительный механизм управления групповой скоростью оптических волновых пакетов в изучаемой неидеальной структуре.

\section{Заключение}

Выполненное в работе изучение зависимости параметров спектра элементарных возбуждений бинарной $1 D$ неидеальной решетки связанных микропор показывает, что в результате внедрения структурных дефектов в массив микропор исследуемой системы можно добиться необходимого изменения энергетической структуры электромагнитных возбуждений и, следовательно, оптических свойств данной системы, обусловленных перестройкой электромагнитного спектра. В частности, выполнено математическое моделирование зависимости дисперсионных характеристик поляритонных возбуждений в неидеальной $1 D$-решетке микропор от концентрации дефектов структуры, связанных с вариацией положений микропор, содержащих в одной из подрешеток квантовые точки. Полученный в работе результат исследования зависимости эффективной массы электромагнитных возбуждений от концентрации структурных дефектов открывает возможность управления групповой скоростью данных элементарных возбуждений, а следовательно, и скоростью передачи сигнала в соответствующем оптоэлектронном устройстве. Таким образом, представ- 
ленные результаты позволяют расширить возможности создания нового класса функциональных пористых материалов - поляритонных систем (массивов микропор, содержащих квантовые точки), позволяющих контролировать распространение электромагнитных возбуждений в таких композитных структурах путем управляемого внедрения в них структурных дефектов.

\section{Финансирование работы}

Исследования выполнены в рамках госбюджетно финансируемого проекта Донецкого физико-технического института им. А.А. Галкина „Формирование структуры и свойств перспективных многофункциональных материалов“.

\section{Конфликт интересов}

Авторы заявляют, что у них нет конфликта интересов.

\section{Список литературы}

[1] Sedov E.S., Alodjants A.P., Arakelian S.M., Chuang Y.-L., Lin Y.Y., Yang W.-X., Lee R.-K. // Phys. Rev. A. 2014. Vol. 89. P. 033828.

[2] Joannopoulos J.D., Johnso S.G., Winn J.N., Meade R.D. Photonic Crystals. Molding the Flow of Light / Second Edition. Princeton: Princeton University Press, 2008. 286 p.

[3] Румянцев В.В., Федоров С.А., Петренко А.Г. // Вестник ДонНУ. Сер. А: Естественные науки. 2017. № 4. С. 69-78.

[4] Kaliteevskii M.A. // Tech. Phys. Lett. 1997. Vol. 23. N 2. P. 120-121.

[5] Vahala K.J. // Nature. 2003. Vol. 424. P. 839-846.

[6] Tighineanu P., Sorensen A.S., Stobbe S., Lodahl P. The Mesoscopic Nature of Quantum Dots in Photon Emission, in: P. Michler (Ed.), Quantum Dots for Quantum Information Technologies. Nano-Optics and Nanophotonics, Cham: Springer, 2017. P. 165-198.

[7] Румянщев В.В., Федоров С.А., Гуменник К.В. // ФТТ. 2017. Т. 59. Вып. 4. С. 741-747.

[8] Rumyantsev V.V., Fedorov S.A., Gumennyk K.V., Gurov D.A., Kavokin A.V. // Superlattices and Microstructures. 2018. Vol. 120. P. 642-649.

[9] Alodjants A.P., Barinov I.O., Arakelian S.M. // J. Phys. B: At. Mol. Opt. Phys. 2010. Vol. 43. P. 095502.

[10] Kasprzak J., Richard M., Kundermann S., Baas A., Jeambrun P., Keeling J.M.J., Marchetti F.M., Szymańska M.H., André R., Staehli J.L., Savona V., Littlewood P.B., Deveaud B., Le Si Dang // Nature. 2006. Vol. 443. P. 409.

[11] Balili R., Hartwell V., Snoke D., Pfeiffer L., West K. // Science. 2007. V. 316. P. 1007.

[12] Amo A., Lefrére J., Pigeon S., Adrados C., Ciuti C., Carusotto I., Houdré R., Giacobino E., Bramati A. // Nature. Phys. 2009. V. 5. P. 805.

[13] Румянщев В.В., Федоров С.А., Гуменник К.В., Гуров Д.А. // ФТВД. 2018. Т. 28. № 1. С. 87-95.

[14] Агранович В.М. Теория экситонов. М.: Наука, 1968. 382 с.

[15] Займан Дж. Модели беспорядка. М.: Мир, 1982. 592 с.

[16] Лось В.Ф. // ТМФ. 1987. Т. 73. № 1. С. 85-102. 